\title{
Reflective and transparent cellulose-based passive radiative coolers
}

\author{
Sampath Gamage • Debashree Banerjee - Md. Mehebub Alam • \\ Tomas Hallberg • Christina Åkerlind · Ayesha Sultana • Ravi Shanker • \\ Magnus Berggren · Xavier Crispin · Hans Kariis • Dan Zhao $\cdot$ Magnus P. Jonsson $(\mathbb{D}$
}

Received: 10 March 2021 / Accepted: 24 July 2021 / Published online: 7 August 2021

(C) The Author(s) 2021

\begin{abstract}
Radiative cooling passively removes heat from objects via emission of thermal radiation to cold space. Suitable radiative cooling materials absorb infrared light while they avoid solar heating by either reflecting or transmitting solar radiation, depending on the application. Here, we demonstrate a reflective radiative cooler and a transparent radiative cooler solely based on cellulose derivatives manufactured via electrospinning and casting, respectively. By modifying the microstructure of cellulose materials, we
\end{abstract}

Debashree Banerjee and Md. Mehebub Alam authors have contributed equally to this work.

Supplementary Information The online version contains supplementary material available at https://doi.org/10.1007/ s10570-021-04112-1.

S. Gamage · D. Banerjee - Md. M. Alam ·

A. Sultana $\cdot$ R. Shanker $\cdot$ M. Berggren

X. Crispin · D. Zhao · M. P. Jonsson ( $\varangle)$

Laboratory of Organic Electronics, Department of Science and Technology, Linköping University,

60174 Norrköping, Sweden

e-mail: magnus.jonsson@liu.se

S. Gamage $\cdot$ D. Banerjee $\cdot$ A. Sultana .

R. Shanker · M. Berggren · X. Crispin - M. P. Jonsson Wallenberg Wood Science Center, Linköping University, 60174 Norrköping, Sweden

T. Hallberg · C. Åkerlind · H. Kariis

Department of Electro-Optical Systems, FOI-Swedish

Defense Research Agency, 58111 Linköping, Sweden control the solar light interaction from highly reflective ( $>90 \%$, porous structure) to highly transparent ( $\approx 90 \%$, homogenous structure). Both cellulose materials show high thermal emissivity and minimal solar absorption, making them suitable for daytime radiative cooling. Used as coatings on silicon samples exposed to sun light at daytime, the reflective and transparent cellulose coolers could passively reduce sample temperatures by up to $15^{\circ} \mathrm{C}$ and $5{ }^{\circ} \mathrm{C}$, respectively.

Keywords Daytime passive radiative cooling . Nanocellulose $\cdot$ Reflective coolers · Transparent coolers $\cdot$ Atmospheric transmittance $\cdot$ Radiative cooling materials

\section{Introduction}

Passive radiative cooling has the potential to reduce the world's energy-consumption by complementing and replacing traditional active methods to control indoor temperature, such as using air-conditioning. In contrast to traditional methods, radiative cooling requires no external energy to function. Instead, it utilizes the temperature difference between the cold outer space and the earth to passively radiate heat through the atmosphere, which conveniently has a transparency window in the infrared (IR) region that 
matches thermal radiation at room temperature. The net cooling power density, $P_{\text {net }}$ of a radiative cooler is given by (see details in Supporting Information):

$P_{n e t}=P_{\text {rad }}-P_{\text {atm }}-P_{\text {nonrad }}-P_{\text {solar }}$

where $P_{\text {rad }}$ is the thermal radiation power per area of the cooler, $P_{\text {atm }}$ is the power per area absorbed by the cooler due to incident radiation from the atmosphere,

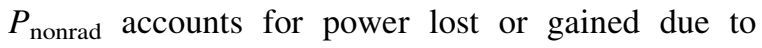
conduction and convection, and $P_{\text {solar }}$ corresponds to incident absorbed power per area due to solar irradiation (Bartoli et al. 1977; Nilsson and Niklasson 1995; Zhao et al. 2019). The last term highlights that efficient radiative cooling is more challenging during daytime than during night time due to solar-induced heating (Bartoli et al. 1977; Nilsson and Niklasson 1995). Daytime radiative coolers therefore need to strongly absorb infrared light (making them strong thermal emitters) while not absorbing light throughout the solar spectrum. Solar radiation must therefore be either transmitted or reflected by the cooling material. Cooling materials that transmit solar radiation are sought for enhancing the performance of solar absorbers (Zhu et al. 2015) and solar cells (Zhu et al. 2014), and have been suggested for cooling objects while preserving their color (Zhu et al. 2013a, b). For many other applications, it is instead critical that solar radiation is reflected by the cooling material to protect underlying objects from absorbing solar light and heating up. Significant effort has been put into creating such reflective radiative coolers, which could cool objects even to sub-ambient temperatures while being exposed to the sun (Rephaeli et al. 2013; Zhai et al. 2017; Li et al. 2019; Mandal et al. 2018; Raman et al. 2014; Chen et al. 2016; Zhou et al. 2019; Kou et al. 2017). These reflective radiative coolers prevent solar transmission using back reflectors (Zhai et al. 2017), non-absorbing visible scatterers (Zhu et al. 2015) or by micro-porous structures (Mandal et al. 2018; Xiang et al. 2020). They are promising as passive techniques to reduce the need for air-conditioning and refrigeration and thereby have potential to decrease energy consumption devoted to cooling (Goldstein et al. 2017). Hence, there is a need for both solar-transparent and solar-reflective radiative coolers, with ideal systems illustrated in Fig. 1a, b, respectively. For transparent coolers we focus on generic systems suitable for different applications, while the transparency window can be further optimized for specific applications, e.g., adopted to the working spectral range of a particular solar cell. Figure 1 shows the ideal reflectance $(R)$, transmittance $(T)$ and absorptance $(A)$ in the UV, visible (VIS) to near IR (NIR) wavelengths and mid-IR (MIR) wavelengths, together with the 1.5 air mass (AM) solar irradiation spectrum (light grey) and the atmospheric IR transparent window (dark grey) from 7 to $14 \mu \mathrm{m}$. An ideal transparent radiative cooler (Fig. 1a) is fully transparent (i.e., no reflection or absorption) in the visible spectral range while an ideal reflective cooler instead reflects all visible light (i.e., no transmission or absorption). In the MIR thermal and atmospheric transparency region, both types of materials must possess high absorptance, which via Kirchoff's law of radiation translates to high thermal emissivity and hence, efficient thermal radiation (Greffet et al. 2018).

While the majority of radiative coolers have been based on inorganic materials, organic materials are emerging as promising alternatives. Not least, cellulose and its derivatives have shown promise by providing both high thermal emissivity and low visible absorption (Li et al. 2019; Xiang et al. 2020; Gamage et al. 2020). Cellulose is an abundant forest-based material with attractive sustainable properties such as biodegradability, biocompatibility and nontoxicity. This makes cellulose-based materials suitable also for passive radiative cooling in human body applications (Wei et al. 2020; Zhang et al. 2019; Peng et al. 2018; Xiao et al. 2019). Furthermore, the visible properties of cellulose materials can be manipulated by additives or via changes in the structural arrangement at the nanoscale and microscale (Vasileva et al. 2017; Koivurova et al. 2018). For example, highly transparent or translucent materials have been reported based on nanofibrillated cellulose (NFC) (Zhu et al. 2013a, b; Fang et al. 2014; Wu et al. 2015; Mahpeykar et al. 2017; Gamage et al. 2020) as well as by delignified and polymer-impregnated wood ( $\mathrm{Li}$ et al. 2016, 2017a, b, 2018). Translucency can be controlled using particle scatterers (Gamage et al. 2020) or by introducing microstructure, which also enables materials to become reflective instead of transparent in the visible range (Xiang et al. 2020). Self-assembly of cellulose nanocrystals into ordered helical structures further allows for materials with structural coloration and preferential reflection of left-handed circularly polarized light (Hewson et al. 2017; Fernandes et al. 2019). 

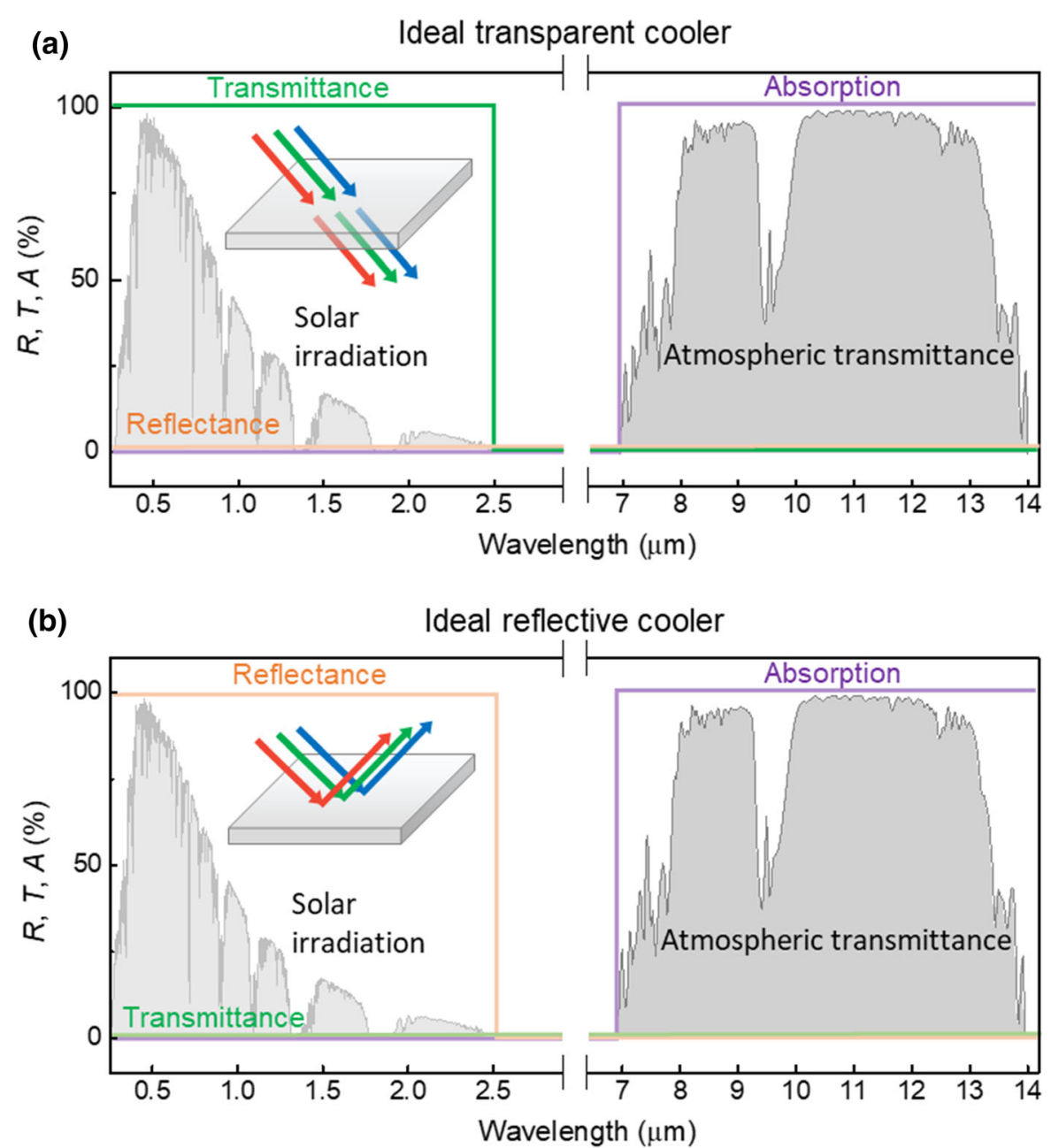

Fig. 1 Optical and mid-IR properties of (a) an ideal transparent radiative cooler and (b) an ideal reflective radiative cooler

Our previous work (Gamage et al. 2020) showed that transparent NFC films can be used for subambient daytime radiative cooling. We also embedded silica microparticles as resonant IR absorbers and visible scatterers to control translucency without compromising cooling performance. Such transparent systems are suitable for coatings on solar cells and other optoelectronic devices, but not to cool objects that need protection from solar radiation and corresponding solar-induced heating. Here, we demonstrate the possibility of creating both reflective and transparent radiative coolers based exclusively on cellulose, without any active additives or back reflectors. The prime difference between the two coolers is the structure of the cellulose at the nano- and microscales. The transparent cooler is homogeneous while the reflective cooler is made porous using an electrospinning process. We characterize the two types of materials in detail using integrating sphere measurements in the UV-VIS-NIR and MIR ranges and compare them in terms of daytime radiative cooling when used as coatings on solar-absorptive silicon substrates.

\section{Experimental}

\section{Materials}

Cellulose Acetate, $(\mathrm{Mn}=30,000)$, Lithium hydroxide (LiOH), N, N-dimethylformamide (DMF) and Glycerol were purchased from Sigma-Aldrich and used as 
received. Carboxymethylated NFC was provided by RISE Bioeconomy.

Electrospun cellulose fiber preparation

A solution of 19 weight percentage (wt $\%$ ) clear cellulose acetate was prepared in co-solvent of DMF and acetone ( $2: 3$ volume ratio) under magnetic stirring at room temperature. As depicted in Fig. 2a, the solution was then sucked into a syringe of needle diameter $0.8 \mathrm{~mm}$ and fed by a syringe pump with a constant flow rate of $10 \mu \mathrm{l} / \mathrm{min}$. A high voltage of $25 \mathrm{kV}$ was maintained between the needle and a collector (aluminum foil) separated by $10 \mathrm{~cm}$ gap during the electrospinning. The prepared fiber mat was then peeled off from the collector and dipped into aqueous solution of $\mathrm{LiOH}(0.1 \mathrm{wt} \%)$ for $8 \mathrm{~h}$ to convert the cellulose acetate to cellulose (see Fig. S1) to obtain a pure cellulose material for suitable comparison with the performance of NFC films. It was then washed 3 times with deionized (DI) water and kept in a fume hood overnight to dry, resulting in a freestanding porous cellulose fiber mat. We maintained the same electrospinning conditions for all samples presented here and obtained different sample thicknesses by varying the fabrication time.

(a)
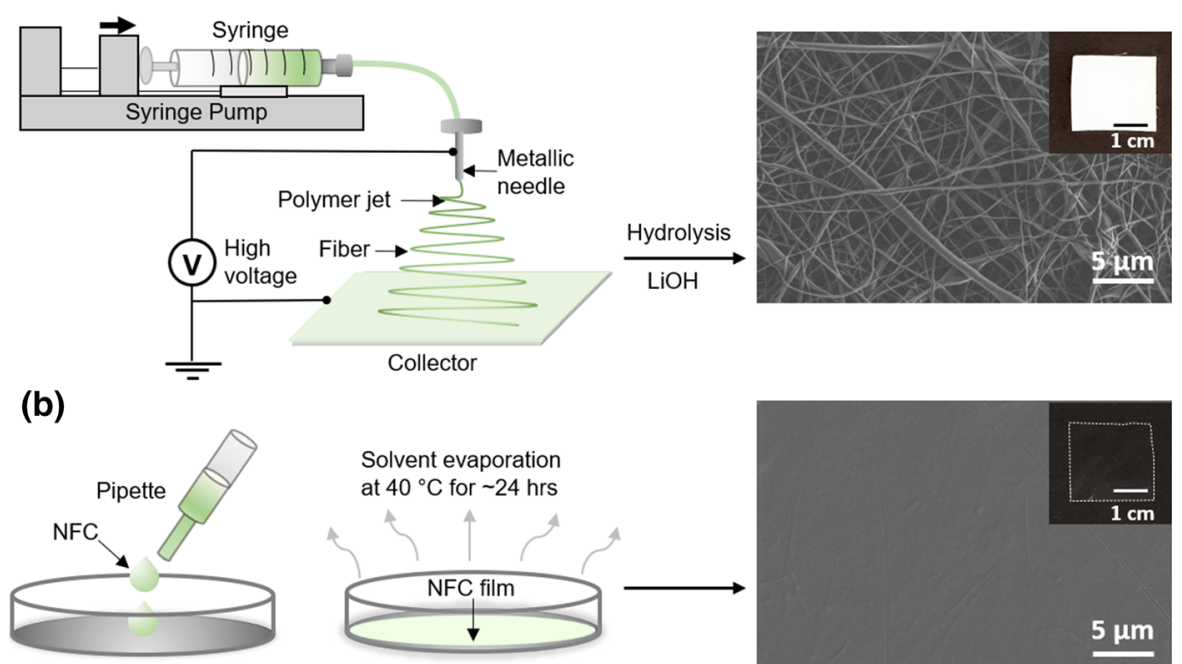

The microstructures of the films were investigated by scanning electron microscopy (SEM), Sigma 500 Gemini from Zeiss AG. Fiber diameter distribution was determined by manual measurements using the line tool of the ImageJ software. For each sample, thickness was measured at 5 different places using a micrometer screw gauge and the average value to the nearest micrometer was taken as the sample thickness.

Reflectance, transmittance and absorptance of samples were determined using spectral directional

The schematic in Fig. 2b depicts the NFC film fabrication casting process. A solution of $0.52 \mathrm{wt} \%$ the NFC solution $10 \%$ glycerol was added, and the final solution was homogenized for 5 min using an ULTRA-TURRAX disperser from IKA Inc. It was then poured into a plastic dish and kept in a drying oven at $40{ }^{\circ} \mathrm{C}$ for about $24 \mathrm{~h}$ in order to dry, resulting in a free-standing transparent cellulose film. To obtain the desired thickness, additional glycerol mixed NFC solution was added to the plastic dish during the drying process.

\section{Sample characterization} samples we

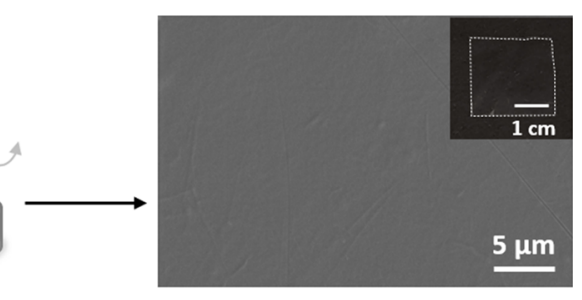

Fig. 2 Schematic illustrations of the processes of preparing (a) porous cellulose by electrospinning, and a SEM image of a resulting film and (b) cellulose films by NFC casting, and a SEM image of a resulting homogenous cellulose film. The insets of

each SEM image show optical images of the porous cellulose (a) and NFC films (b), respectively. The boundary of the NFC film in the optical image is marked with a dashed line as a guide to the eye 
hemispherical reflectance (DHR) and directional hemispherical transmittance (DHT). Two different spectrometers were used to cover the wavelength regions from the UV to the far IR: A Cary 5000 in the region 250-2500 $\mathrm{nm}$ and a Bruker Vertex 70 Fourier Transform Infrared (FTIR) spectrometer for the region 2-33 $\mu \mathrm{m}$. Both instruments were equipped with integrating spheres illuminating the sample at an angle of incidence $\left(\theta_{i}\right)$ of $8^{\circ}$ and $9^{\circ}$, for the Cary and Bruker spectrometers, respectively. A DRA-2500 integrating sphere from Labsphere was used for the Cary spectrophotometer and a Labsphere A562 was used for the Bruker FTIR. The following detectors were used for the different spectral ranges: R928 PMT for UV-Vis, a cooled PbS for NIR up to $2500 \mathrm{~nm}$ and a DTGS detector in the IR.

For reflectance measurements, both instruments make use of calibrated reflectance standards (Spectralon ${ }^{\circledR}$ and Infragold ${ }^{\circledR}$ calibrated standards from Labsphere). For the Cary instrument, the reflectance standard was used to collect the baseline, which is used when calculating the sample DHR. The integrating sphere of the Bruker FTIR makes use of an absolute reflection method using the interior wall of the sphere for the baseline measurement, but where the results of the DHR are corrected by a factor obtained from measurements on different calibrated reflectance standards to ensure accurate measurement results. The spectral absorptance is then obtained from the spectral reflectance and transmittance via:

$R(\lambda)+T(\lambda)+A(\lambda)=1$

with $R, T$ and $A$ being in the range $0-1$ (or $0-100 \%$ ). We note here that our measurements (DHR and DHT) accounts for diffusive reflection and transmission and not only specular reflection and direct transmission. By Kirchhoff's law of radiation the emissivity at any given wavelength $(\varepsilon(\lambda))$ for a surface in thermal equilibrium is then given by:

$\varepsilon(\lambda)=A(\lambda)=1-T(\lambda)-R(\lambda)$

\section{Radiative cooling measurements}

The samples were placed in a line in the middle of a measurement box of around $40 \times 30 \times 15 \mathrm{~cm}$. A thin polyethylene (PE) sheet was used to cover the measurement box in order to suppress temperature fluctuations due to wind disturbances. Two thermocouples were used to measure the air temperature inside the box and the average of those two measurements was taken as the ambient temperature. These two thermocouples were shaded from direct sun light as well as exposure to the sky by a dual shade by an Almylar-covered cardboard piece and a folded white cloth. Each cooler was stuck to a silicon wafer using stripes of double-sided adhesive tape (around $1 \mathrm{~mm}$ wide) at the edges to ensure that most of the bottom surface area of the cooler was in direct contact with the $\mathrm{Si}$ wafer. Real-time temperature values of the thermocouples were recorded using a LabVIEW program interfaced by a voltage to temperature conversion interface and an Arduino processor. The radiative cooling measurements were performed in Norrköping, Sweden on June 18, 2021 (unless otherwise stated), with a maximum outdoor temperature of around $36{ }^{\circ} \mathrm{C}$.

\section{Results and discussion}

Figure 2a and $\mathrm{b}$ show the processes and SEM images of cellulose films made by electrospinning and casting, respectively. The two fabrication methods result in highly different structure at the microscale. The electrospun cellulose film is highly porous, composed of a scaffold network of cellulose fibers with microand nanoscale dimensions. By contrast, the casted NFC film is highly homogenous. The microporous structure of the electrospun film leads to broadband scattering in the visible region, which makes the film white, in stark contrast to the transparent casted NFC film (see inset photographs in Fig. 2a and b). Successful conversion of the cellulose acetate to cellulose was verified via changes in the vibrational peaks of FTIR spectra before and after conversion (Huang et al. 2015), as shown in Fig. S1. Examining SEM images for converted cellulose samples indicates a unimodal fiber diameter distribution with a peak around $175 \mathrm{~nm}$ (Fig. S2).

Detailed studies of the reflectance, transmittance and absorptance of the two different cellulose films confirm our initial indications based on visual appearance. As shown in Fig. 3a (blue line), the porous cellulose material system is exceptionally reflective in the visible, with average $94 \%$ reflectance in the range from 0.4 to $0.7 \mu \mathrm{m}$ for a $275 \mu \mathrm{m}$ thick sample. The 

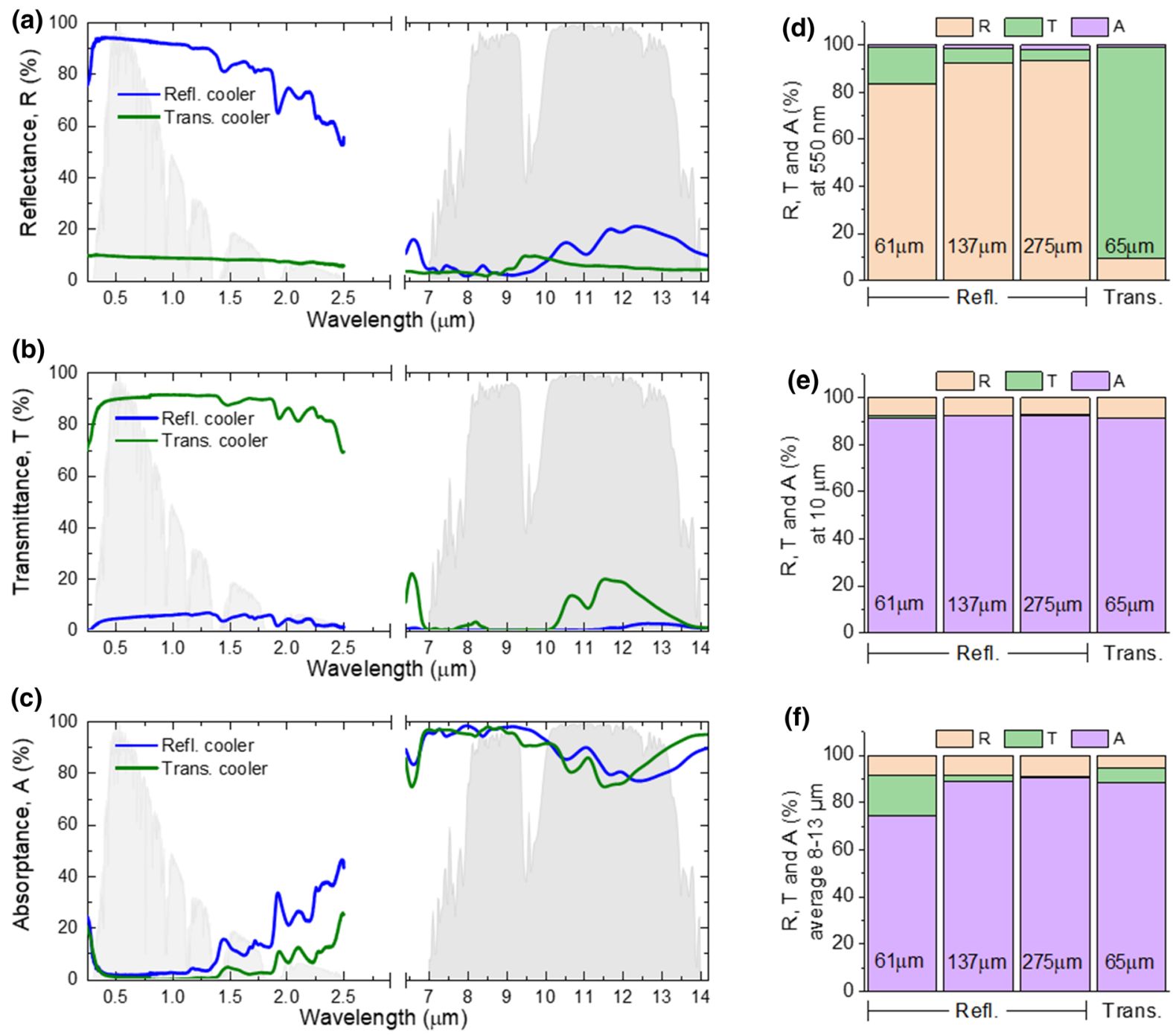

Fig. 3 Optical and MIR properties of reflective and transparent coolers. Comparison of a reflectance, $R, \mathbf{b}$ transmittance, $T$ and c absorptance, $A$ of a reflective cooler (thickness $275 \mu \mathrm{m}$ ) and a transparent cooler (thickness $65 \mu \mathrm{m}$ ). The grey background spectra in (a-c) correspond to the solar irradiance (light grey)

reflectance decreases in the UV but remains above $75 \%$ for wavelengths down to $0.25 \mu \mathrm{m}$. Likewise, the reflectance slowly decreases but remains fairly high at longer wavelengths all the way to $2.5 \mu \mathrm{m}$. The film shows less than $10 \%$ transmittance in the whole UVVis-NIR region $(0.25-2.5 \mu \mathrm{m}$, Fig. 3b), thereby efficiently preventing light absorption by underlying objects when used as a coating. The broadband high reflection is due to scattering by the wide distribution of nano- and microstructures in the porous material. By contrast, a casted homogenous cellulose film and atmospheric transmittance (dark grey) in arbitrary units. $R$, $T$ and $A$ of three reflective coolers (thicknesses 61, 137 and $275 \mu \mathrm{m})$ and for a $65 \mu \mathrm{m}$ thick transparent cellulose cooler (d) at $550 \mathrm{~nm}$, (e) at $10 \mu \mathrm{m}$ and (f) averaged in the range 8-13 $\mu \mathrm{m}$ of the atmospheric transparent window

(green line in Fig. 3b, $65 \mu \mathrm{m}$ thick) provides high transmittance and less than $10 \%$ reflectance (Fig. 3a) in the whole UV-Vis-NIR region $(0.25-2.5 \mu \mathrm{m})$. Based on this marked difference, we now denote the electrospun porous cellulose films as reflective coolers and the homogenous casted films as transparent coolers. Importantly, both types of coolers show very low absorptance from 0.3 to $1.3 \mu \mathrm{m}$ (Fig. 3c), which is a promising feature to avoid solar heating of the cooling materials. The absorptance increases towards the UV region and at longer wavelengths in the NIR, 
but in terms of solar heating we note that solar irradiation is also lower in these regions (indicated by the light-grey shaded spectrum). The increase in absorptance at longer wavelengths is more pronounced for the reflective cooler, which may be due to the high microporosity and differences in film thickness.

While both types of materials show low absorptance in the visible, they instead show high absorptance in the mid-IR region (Fig. 3c). As discussed above, these combined absorptance characteristics makes the materials suitable for daytime passive radiative cooling. From 7 to $10 \mu \mathrm{m}$, both coolers are almost opaque. The transmittance of the reflective cooler further remains very low at longer MIR wavelengths while the transparent cooler shows a broad peak at around $12 \mu \mathrm{m}$ with about $20 \%$ transmittance. Comparison between additional samples reveal that this difference in MIR transmittance between the samples is related to differences in film thickness and porosity, as investigated next.

Figure $3 \mathrm{~d}-\mathrm{f}$ summarizes the visible and MIR properties for three reflective cellulose coolers of different thicknesses $(61,137$ and $275 \mu \mathrm{m})$ and for the $65 \mu \mathrm{m}$ thick transparent cellulose cooler. Since the visible reflection, transmission and absorption were relatively flat, we present the data at a representative wavelength of $550 \mathrm{~nm}$ (Fig. 3d). All three reflective coolers show very low absorption $(<3 \%)$ in the visible region. In terms of reflection, even the thinnest reflective cooler provides values higher than $80 \%$. This value increases with thickness, reaching above 93\% for the thickest reflective cooler. The gradual increase in reflection with thickness can be explained by additional scattering sites for thicker films and corresponding increased probability for light to back scatter instead of escaping the film in the forward direction. Further increasing the electrospun thickness while maintaining film uniformity is challenging because the high voltage applied between needle and collector becomes less effective when more material is deposited on the collector. Figure $3 e$ presents the equivalent summary of the MIR properties of the same samples. We first use $10 \mu \mathrm{m}$ as representative wavelength at which the thermal emission is peaking at room temperature. All four coolers are essentially opaque at this wavelength, with around 90\% absorption and around $10 \%$ reflection. Since the MIR atmospheric transparent window spans from about 8 to $13 \mu \mathrm{m}$ and thermal radiation is spectrally broad, we also present the average MIR properties for the four coolers in this wavelength range (Fig. 3f). This analysis reveals larger variations between the samples. We first note that the thinnest reflective cooler shows lower absorptance (i.e., lower emissivity) and higher transmittance in the MIR compared with the transparent cooler despite very similar thicknesses, which can be explained by lower density and less material in the beam path for the porous material. We further observe that increasing the thickness of the reflective cellulose cooler helps to improve the MIR absorptance and thermal emissivity to around $90 \%$. The merely $65 \mu \mathrm{m}$ thick transparent cooler also shows high (>85\%) averaged MIR absorption in the atmospheric window, verifying that both types of materials can provide high thermal emissivity at room temperature.

From the data presented in Fig. 3, we conclude that both types of cellulose materials possess very low absorptance in the visible range while being highly absorptive in the MIR region and thereby efficient thermal emitters. These results suggest that both types of materials are suitable for daytime radiative cooling. We performed real-time outdoor radiative cooling measurements during daytime with reflective and transparent coolers. The sky was clear with no clouds, with effective solar irradiance of around 0.8 sun during the measurement and relative humidity of around $27 \%$. The coolers were placed in a cardboard box with bottom and side surfaces covered with reflective metalized mylar sheets (see Fig. 4a). Convective heat transfer was lowered using an IR-transparent polyethylene film as wind barrier. We placed foam blocks wrapped with metalized mylar sheets at the bottom of the box and silicon wafers on top of these blocks representing objects to be cooled (and protected from solar heating, see Fig. 4b). The optical properties of the silicon substrate are given in Fig. S3. The reflective and transparent cellulose radiative coolers were then used as coatings on these silicon wafers while monitoring their temperatures during exposure to the sun and the sky. The top panel of Fig. 4c shows the temperature variation during such measurements for silicon wafers coated with a reflective cellulose cooler ( $275 \mu \mathrm{m}$ thick, blue), a transparent cellulose cooler (65 $\mu \mathrm{m}$ thick, green), a non-coated silicon substrate (dark grey) and the ambient temperature in the measurement box (light grey). The ambient temperature is presented as the average between two shaded 
(a)

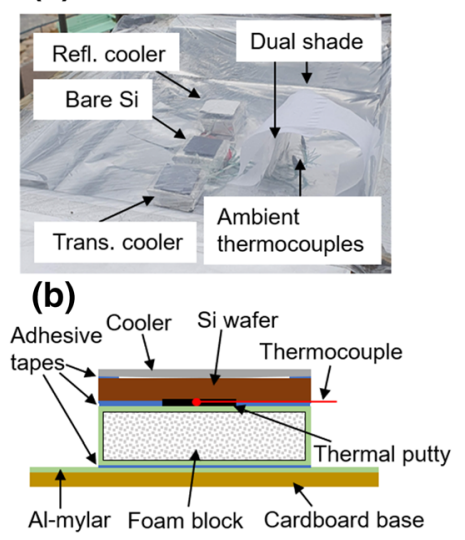

Fig. 4 Cooling measurements of transparent and reflective coolers as coatings on silicon substrates. a Photograph of the temperature measurement box with the two coolers on silicon, a bare silicon wafer and the shaded thermocouples measuring the ambient temperature. $\mathbf{b}$ Schematic cross-section of a radiative cooler arrangement for measurements with thermocouple and

thermocouples in the box. The temperature of the bare silicon wafer reached around $68{ }^{\circ} \mathrm{C}$ while the wafers coated with the cellulose coolers both maintained lower temperatures (around $63{ }^{\circ} \mathrm{C}$ and $53{ }^{\circ} \mathrm{C}$ for the transparent and reflective coolers, respectively). The elevated temperature in the box compared with the outdoor temperature (up to around $36{ }^{\circ} \mathrm{C}$ ) is due to solar absorption and heat generation of components in the box, including the bare silicon substrate. The reduction in temperature by the cellulose coolers ( $\Delta T=T_{\mathrm{Si}}-T_{\text {cooler }}$ ) is presented in the bottom panel of Fig. 4c. The transparent radiative cooler showed $\Delta T \approx 5{ }^{\circ} \mathrm{C}$, despite allowing most of the solar radiation to reach (and heat up) the wafer. The absolute temperatures varied from day to day depending on, for example, weather conditions, and we observed transparent cooler with temperature reduction even up to $15^{\circ} \mathrm{C}$ (Fig. S4). Similar cooling performance of $14{ }^{\circ} \mathrm{C}$ relative to bare silicon has been reported for a cooler based on $\mathrm{SiO}_{2}$ microspheres on glass (Jaramillo-Fernandez et al. 2019) and the temperature reductions are significant compared to the theoretically suggested value of $18.3 \mathrm{~K}$ for transparent coolers (Zhu et al. 2014). The reflective cooler reduced the temperature more than the transparent cooler and continuously managed to maintain temperatures several degrees Celsius below the ambient air temperature inside the box. The reason for the more efficient temperature reduction (around $15{ }^{\circ} \mathrm{C}$ for the (c)

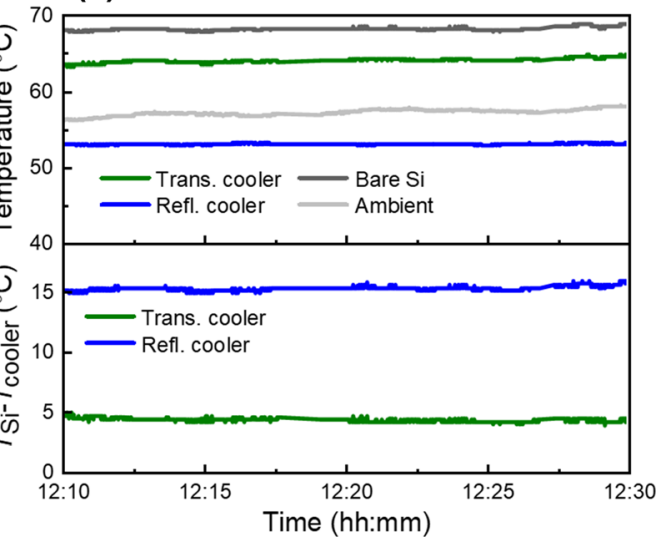

insulating materials. c Temperature measurement of two coolers and a bare silicon wafer during daytime on a sunny day with clear sky. The reduction in temperature of the two types of coolers relative to the temperature of the bare silicon wafer is presented in the bottom panel

measurement in Fig. 4) is that the reflective cellulose cooler also suppresses solar-induced heating of the underlying silicon wafer. Such sub-ambient daytime cooling highlights the dual ability of the reflective cooler to both prevent solar heating and also remove heat by radiative cooling. While absolute temperatures varied between measurements, the qualitative differences between the two types of coolers were consistent. To further understand the functions of the transparent and reflective coolers (both on silicon) we estimated their net cooling power density by calculating the different terms in Eq. 1 (see Supporting Information for details). Figure S7 present the results for different ambient temperatures, sample temperatures and nonradiative heat transfer conditions. The results predict the transparent cooler to provide negative net cooling power density at $T_{\text {cooler }}=$ $T_{\text {ambient }}$ for all investigated conditions, which explains why the transparent cooler only shows reduction in temperature from the bare silicon substrate reduction but not sub-ambient cooling. By contrast, the reflective cooler provides positive net cooling power density at $T_{\text {cooler }}=T_{\text {ambient }}$, corroborating its ability for subambient cooling thanks to additional suppression of solar absorption.

To avoid effects from solar heating, we also performed measurements during nighttime using the same coolers. As expected, these results present subambient cooling also for the transparent cooler 
(Fig. S5). Furthermore, we note that also the ambient temperature in the box decreased significantly $(\approx$ $4{ }^{\circ} \mathrm{C}$ ) when the setup was exposed to the sky. This interesting aspect may be due to the coolers also cooling the region where the ambient temperature was measured. In turn, this means that the cooling performance may be underestimated when the temperatures of the coolers are compared with the measured ambient temperature in the box. We finally present results for a daytime measurement without the IR transparent wind barrier (Fig. S6). Also here, both coolers could significantly reduce the temperature of the underlying silicon wafers compared with the noncoated silicon, with the reflective cooler being most effective. These measurements without the wind barrier also showed overall lower temperatures compared with corresponding measurements with wind barrier, as attributed to additional convective heat removal for the open system.

\section{Conclusion}

Our study shows that cellulose is suitable for passive radiative cooling and that different types of coolers can be made by varying the preparation method. We formed homogenous cellulose films by casting and porous cellulose fiber network films by electrospinning. Even though both systems are cellulose-based, they demonstrate opposite visible reflection/transmission behavior due their structural differences at the nano- and microscales. They both further show very low solar absorptance combined with high thermal emissivity in the MIR. The average MIR absorptance, and hence thermal emissivity, of both types of materials was greater than $74 \%$ for all investigated sample thicknesses and reached up to $90 \%$ for the thickest reflective cooler. Daytime radiative cooling measurements demonstrate that the reflective and transparent coolers could reduce the temperature of coated silicon wafers by around $15^{\circ} \mathrm{C}$ and $5{ }^{\circ} \mathrm{C}$, respectively. Moreover, the reflective cooler reached a few degrees Celsius even below the ambient temperature. These results suggest that transparent cellulose may be used as coating materials for optoelectronic and solar harvesting devices to reduce heat-induced performance drops, while reflective cellulose shows promise as coatings for cooling other underlying objects, such as water masses for building space cooling systems. To ensure durability and weather resistance of the coolers without compromising their optical characteristics, future work may also employ thin transparent lamination layers, for example, of polyethylene as is now used to cover the measurement box.

Acknowledgments The authors acknowledge financial support from the Knut and Alice Wallenberg Foundation via a Wallenberg Scholarship; the Knut and Alice Wallenberg foundation, Linköping University and industry through the Wallenberg Wood Science Center; the Swedish Government Strategic Research Area in Materials Science on Functional Materials at Linköping University (Faculty Grant SFO-Mat-LiU No. 2009 00971), the Swedish Foundation for Strategic Research and the Swedish Armed Forces Research and Technology program and the Swedish Research Council (VR 2016-06146). The authors also thank Meysam Karami Rad for assistance with constructing the temperature measurement setup.

Funding Open access funding provided by Linköping University.

\section{Declarations}

Conflict of interest The authors have no conflict of interests.

Ethical approval The study was completed by following ethical standards.

Open Access This article is licensed under a Creative Commons Attribution 4.0 International License, which permits use, sharing, adaptation, distribution and reproduction in any medium or format, as long as you give appropriate credit to the original author(s) and the source, provide a link to the Creative Commons licence, and indicate if changes were made. The images or other third party material in this article are included in the article's Creative Commons licence, unless indicated otherwise in a credit line to the material. If material is not included in the article's Creative Commons licence and your intended use is not permitted by statutory regulation or exceeds the permitted use, you will need to obtain permission directly from the copyright holder. To view a copy of this licence, visit http://creativecommons.org/licenses/by/4.0/.

\section{References}

Bartoli B, Catalanotti S, Coluzzi B, Cuomo V, Silvestrini V, Troise G (1977) Nocturnal and diurnal performances of selective radiators. Appl Energy. https://doi.org/10.1016/ 0306-2619(77)90015-0

Chen Z, Zhu L, Raman A, Fan S (2016) Radiative cooling to deep sub-freezing temperatures through a 24-h day-night cycle. Nat Commun. https://doi.org/10.1038/ ncomms 13729 
Fang Z, Zhu H, Yuan Y, Ha D, Zhu S, Preston C, Chen Q et al (2014) Novel nanostructured paper with ultrahigh transparency and ultrahigh haze for solar cells. Nano Lett. https://doi.org/10.1021/nl404101p

Fernandes SN, Lopes LF, Godinho MH (2019) Recent advances in the manipulation of circularly polarised light with cellulose nanocrystal films. Curr Opin Solid State Mater Sci. https://doi.org/10.1016/j.cossms.2018.11.004

Gamage S, Kang ESH, Åkerlind C, Sardar S, Edberg J, Kariis H, Ederth T, Berggren M, Jonsson MP (2020) Transparent nanocellulose metamaterial enables controlled optical diffusion and radiative cooling. J Mater Chem C. https:// doi.org/10.1039/d0tc01226b

Goldstein EA, Raman AP, Fan S (2017) Sub-ambient nonevaporative fluid cooling with the sky. Nat Energy. https:// doi.org/10.1038/nenergy.2017.143

Greffet JJ, Patrick B, Giovanni B, Emilie S, François M (2018) Generalized kirchhoff law. https://doi.org/10.1103/ PhysRevX.8.021008

Hewson D, Vukusic P, Eichhorn SJ (2017) Reflection of circularly polarized light and the effect of particle distribution on circular dichroism in evaporation induced self-assembled cellulose nanocrystal thin films. AIP Adv. https://doi. org/10.1063/1.4986761

Huang F, Yunfei Xu, Peng B, Yangfen Su, Jiang F, Hsieh YL, Wei Q (2015) Coaxial electrospun cellulose-core fluoropolymer-shell fibrous membrane from recycled cigarette filter as separator for high performance lithium-ion battery. ACS Sustain Chem Eng. https://doi.org/10.1021/ acssuschemeng.5b00032

Jaramillo-Fernandez J, Whitworth GL, Pariente JA, Blanco A, Garcia PD, Lopez C, Sotomayor-Torres CM (2019) A SelfAssembled 2D thermofunctional material for radiative cooling. Small. https://doi.org/10.1002/smll.201905290

Koivurova M, Vasileva E, Li Y, Berglund L, Popov S (2018) Complete spatial coherence characterization of quasi-random laser emission from dye doped transparent wood. Opt Exp. https://doi.org/10.1364/oe.26.013474

Kou Jun Long, Jurado Zoila, Chen Zhen, Fan Shanhui, Minnich Austin J (2017) Daytime radiative cooling using near-black infrared emitters. ACS Photon. https://doi.org/10.1021/ acsphotonics.6b00991

Leroy A, Bhatia B, Kelsall CC, Castillejo-Cuberos A, Di Capua MH, Zhao L, Zhang L, Guzman AM, Wang EN (2019) High-performance subambient radiative cooling enabled by optically selective and thermally insulating polyethylene aerogel. Sci Adv. https://doi.org/10.1126/sciadv. aat9480

Li Y, Qiliang Fu, Shun Yu, Yan M, Berglund L (2016) Optically transparent wood from a nanoporous cellulosic template: combining functional and structural performance. Biomacromol. https://doi.org/10.1021/acs.biomac. $6 \mathrm{~b} 00145$

Li Y, Qiliang Fu, Rojas R, Yan M, Lawoko M, Berglund L (2017a) Lignin-retaining transparent wood. Chemsuschem. https://doi.org/10.1002/cssc.201701089

Li Y, Shun Yu, Veinot JGC, Linnros J, Berglund L, Sychugov I (2017b) Luminescent transparent wood. Adv Opt Mater. https://doi.org/10.1002/adom.201600834

Li Y, Qiliang F, Yang X, Berglund L (2018) Transparent wood for functional and structural applications. Philos Trans R
Soc A Math Phys Eng Sci. https://doi.org/10.1098/rsta. 2017.0182

Li T, Zhai Y, He S, Gan W, Wei Z, Heidarinejad M, Dalgo D et al (2019) A radiative cooling structural material. Science. https://doi.org/10.1126/science.aau9101

Mahpeykar SM, Zhao YB, Li XY, Yang ZY, Xu QW, Lu ZH, Sargent EH, Wang XH (2017) Cellulose nanocrystal: polymer hybrid optical diffusers for index-matching-free light management in optoelectronic devices. Adv Opt Mater. https://doi.org/10.1002/adom.201700430

Mandal J, Yanke Fu, Overvig AC, Jia M, Sun K, Shi NN, Zhou H, Xiao X, Nanfang Yu, Yang Y (2018) Hierarchically porous polymer coatings for highly efficient passive daytime radiative cooling. Science. https://doi.org/10.1126/ science.aat9513

Nilsson TMJ, Niklasson GA (1995) Radiative cooling during the day: simulations and experiments on pigmented polyethylene cover foils. Sol Energy Mater Sol Cells. https:// doi.org/10.1016/0927-0248(94)00200-2

Peng Y, Chen J, Song AY, Catrysse PB, Hsu PC, Cai L, Liu B et al (2018) Nanoporous polyethylene microfibres for large-scale radiative cooling fabric. Nat Sustain. https:// doi.org/10.1038/s41893-018-0023-2

Raman AP, Anoma MA, Zhu L, Rephaeli E, Fan S (2014) Passive radiative cooling below ambient air temperature under direct sunlight. Nature 515(7528):540-544. https:// doi.org/10.1038/nature13883

Rephaeli E, Raman A, Fan S (2013) Ultrabroadband photonic structures to achieve high-performance daytime radiative cooling. Nano Lett. https://doi.org/10.1021/n14004283

Vasileva E, Li Y, Sychugov I, Mensi M, Berglund L, Popov S (2017) Lasing from organic dye molecules embedded in transparent wood. Adv Opt Mater. https://doi.org/10.1002/ adom.201700057

Wei W, Zhu Y, Li Q, Cheng Z, Yao Y, Zhao Qi, Zhang P et al (2020) An $\mathrm{Al}_{2} \mathrm{O}_{3}$-cellulose acetate-coated textile for human body cooling. Sol Energy Mater Sol Cells. https:// doi.org/10.1016/j.solmat.2020.110525

Wu W, Tassi NG, Zhu HL, Fang ZQ, Hu LB (2015) Nanocellulose-based translucent diffuser for optoelectronic device applications with dramatic improvement of light coupling. ACS Appl Mater Interfaces 7(48):26860-26864. https:// doi.org/10.1021/acsami.5b09249

Xiang B, Zhang R, Luo Y, Zhang S, Lei X, Min H, Tang S, Meng X (2020) 3D porous polymer film with designed pore architecture and auto-deposited sio2 for highly efficient passive radiative cooling. Nano Energy. https://doi.org/10. 1016/j.nanoen.2020.105600

Xiao R, Hou C, Yang W, Yun Su, Li Y, Zhang Q, Gao P, Wang $\mathrm{H}$ (2019) Infrared-radiation-enhanced nanofiber membrane for sky radiative cooling of the human body. ACS Appl Mater Interfaces. https://doi.org/10.1021/acsami.9b13933

Zhai Y, Ma Y, David SN, Zhao D, Lou R, Tan G, Yang R, Yin X (2017) Scalable-manufactured randomized glass-polymer hybrid metamaterial for daytime radiative cooling. Science 355(6329):1062-1066. https://doi.org/10.1126/science. aai7899

Zhang XuA, Shangjie Yu, Beibei Xu, Li M, Peng Z, Wang Y, Deng $S$ et al (2019) Dynamic gating of infrared radiation in a textile. Science. https://doi.org/10.1126/science.aau1217 
Zhao D, Aili A, Zhai Y, Shaoyu Xu, Tan G, Yin X, Yang R (2019) Radiative sky cooling: fundamental principles, materials, and applications. Appl Phys Rev. https://doi.org/ 10.1063/1.5087281

Zhou L, Song H, Liang J, Singer M, Zhou M, Stegenburgs E, Zhang N et al (2019) A polydimethylsiloxane-coated metal structure for all-day radiative cooling. Nat Sustain. https:// doi.org/10.1038/s41893-019-0348-5

Zhu H, Parvinian S, Preston C, Vaaland O, Ruan Z, Liangbing $\mathrm{Hu}$ (2013a) Transparent nanopaper with tailored optical properties. Nanoscale. https://doi.org/10.1039/c3nr00520h

Zhu L, Raman A, Fan S (2013b) Color-preserving daytime radiative cooling. Appl Phys Lett. https://doi.org/10.1063/ 1.4835995
Zhu L, Raman A, Wang KX, Anoma MA, Fan S (2014) Radiative cooling of solar cells. Optica. https://doi.org/10. 1364/optica.1.000032

Zhu L, Raman AP, Fan S (2015) Radiative cooling of solar absorbers using a visibly transparent photonic crystal thermal blackbody. Proc Natl Acad Sci USA. https://doi. org/10.1073/pnas.1509453112

Publisher's Note Springer Nature remains neutral with regard to jurisdictional claims in published maps and institutional affiliations. 